\title{
A SUBGROUP METHOD BASED ON THE EQUIVALENT DANCOFF-FACTOR CELL TECHNIQUE COMPARED WITH THE FINE-STRUCTURE METHOD IN APOLLO3 ${ }^{\circledR}$
}

\author{
Li Mao*, Igor Zmijarevic, Richard Sanchez and Nicolas Gerard Castaing \\ DEN-Service d'études des réacteurs et de mathématiques appliquées (SERMA), CEA, \\ Université Paris-Saclay, F-91191 Gif-sur-Yvette, France
}

li.leimao@cea.fr, igor.zmijarevic@cea.fr, richard.sanchez@cea.fr, nicolas.gerardcastaing@cea.fr

\begin{abstract}
We compare a newly implemented subgroup method, which is based on the equivalent Dancoff-factor cell technique, with the fine structure method in APOLLO3 ${ }^{\circledR}$. The new method obtains precise reaction rates and consequently is precise in predicting multiplication factor. It can reduce the time in the self-shielding calculation by a factor of 38 compared with the subgroup method based on the multi-cell approximation in a PWR Gd-UOX assembly calculation. The fine structure method obtains larger errors in reaction rates than the subgroup method, but the error compensation sometimes leads to small errors in multiplication factors. This work demonstrates the precision and the efficiency of the new method.
\end{abstract}

KEYWORDS: resonance self-shielding, subgroup method, SPH method, equivalent Dancoff-factor cell method, fine structure method

\section{INTRODUCTION}

Resonance self-shielding is a primary step in multigroup lattice calculations. The precision of the self-shielding method determines the precision of lattice calculations and subsequent core calculations. The fine-structure method (FSM) in APOLLO ${ }^{\circledR}[1]$, has been developed based on the FSM of the APOLLO2 code [2]. The method performs the self-shielding calculations by solving the fine-structure equation.

The FSM combines the subgroup method with the equivalence theory, in order to use a coarse energy mesh and the mathematical probability tables that preserve the moments of point-wise cross sections. With the coarse group grid, the subgroup assumption that the cross sections are not correlated with the source term in a group, is not verified in the resolved resonance domain.

The remedy adopted by the FSM is the double equivalences: (1) The heterogeneous - homogeneous equivalence: the heterogeneous equation is first solved by using the subgroup method. We obtain

\footnotetext{
${ }^{*}$ Corresponding author, Tel: +33(0)1 69087051
} 
the equivalent dilutions per region, per group and per resonant isotope by preserving the reaction rates. The reference reaction rates are then interpolated with the dilutions in the pre-tabulated reaction rates of infinite homogeneous media; (2) The multigroup equivalence: we calculate the self-shielded cross sections by iterations to preserve the reference reaction rates. The FSM obtains rather good precision, with a discrepancy of less than $300 \mathrm{pcm}$ in multiplication factors in thermal reactor calculations. However it has difficulties to achieve a better precision.

The issue of the angular dependence of multigroup cross sections in resonance self-shielding was revealed recently [3]. The direct integration of the continuous-energy transport equation over an energy group results in a multigroup equation with angular-dependent total cross sections. Using the cross sections condensed by Monte Carlo scalar flux leads to an error around $-200 \mathrm{pcm}$ in the multiplication factor of a PWR UOX pin-cell [4]. The remedy is to apply the superhomogenization (SPH) method, which was originally introduced to preserve the reaction rates in spatial homogenization [5]. In the example above, the discrepancy in the multiplication factor could be reduced to less than $10 \mathrm{pcm}$, if we apply the SPH technique [4].

In the FSM, the multigroup equivalence, which is similar to the SPH, is performed. However, we still have $-200 \mathrm{pcm}$ to $-300 \mathrm{pcm}$ discrepancies in multiplication factors with the FSM. Both multigroup equivalence and SPH compute the cross sections by preserving the reference reaction rates. If the cross sections obtained by the multigroup equivalence can not reproduce the reference results, we believe that the reference reaction rates are not correct. In fact, in order to fix the slowing-down bias due to the use of a coarse energy mesh, the heterogeneous reaction rates are replaced by those of the infinite homogeneous media in the FSM. In consequence, the actual spatial reaction rates are lost in the heterogeneous-homogeneous equivalence.

To remedy this problem, we have developed a new subgroup method based on SPH technique (SG+SPH) [4] in APOLLO3. This method employs the LWR 383-group mesh, which is fine enough to avoid the heterogeneous-homogeneous equivalence. It employs the same Improved Direct Method (IDM) [1,4] of the FSM to solve the subgroup fine-structure equation. Then the $\mathrm{SPH}$ is carried out to obtain the cross sections that preserve the reaction rates. The IDM method is capable of treating resonant up-scattering and resonance interference, which are the two primary phenomena to be considered in the self-shielding calculations.

Our preliminary tests showed that the SG+SPH method was precise in the calculations of PWR cells and assemblies with UOX, MOX, and Gd-UOX fuels: the errors in multiplication factors are less than $50 \mathrm{pcm}$ compared with the Monte Carlo references. However, the new method needs a long execution time in assembly calculations, due to the mixture treatment and the assembly collision probability calculation. When the number of mixtures increases, the number of self-shielding calculations increases proportionally, so does the number of calculations of assembly collision probabilities. In a depletion calculation of an eighth $17 \times 17$ fuel assembly, which has 45 pins of the same fuel material being divided into six rings, the number of mixtures can increase from one up to 270 as the burnup increases. Thus the number of the collision probability calculations with the multi-cell approximation (CP-MCA) at a burnup step can be up to 270 times the number at zero-burnup. Since the time for one assembly CP-MCA calculation is not trivial, this time increase becomes excessive for a practical calculation.

To remedy this issue, an Equivalent Dancoff Cell (EDC) method, originally proposed in [6], has 
been recently implemented in APOLLO3 [7]. With the EDC method, we carry out only once the assembly CP-MCA calculation, by setting the fuel cross sections to $10^{5} \mathrm{~cm}^{-1}$ and those of moderator and cladding to potential cross sections. Then the Dancoff factors of fuel pins are calculated using the assembly collision probabilities. For each fuel pin, an associated one-dimensional (1D) cylindrical cell, an EDC, is calculated to have the external radius determined by preserving the Dancoff factor in the two-dimensional (2D) geometry. Finally, the self-shielding calculation is only needed to be applied to the EDCs.

The preliminary tests showed that the EDC method gives similar results as the MCA method, the differences in multiplication factor of the two methods are generally less than $15 \mathrm{pcm}$. However, the EDC method can reduce the self-shielding time of an assembly by a factor of 16 to 53 compared with the MCA method [7]. In this paper, we will compare the calculations of fuel cells and assemblies by the EDC method and the FSM. Section 2 presents a brief resume of the theory of the EDC-based SG+SPH method. Section 3 gives numerical results. The conclusions are given in the end.

\section{THEORY}

The SG+SPH method was presented in detail in our paper [4] and the EDC method implemented in APOLLO3 was described in Ref. [7]. Here we give a brief review of the theory.

\subsection{The Subgroup Method Based on the Fine-Structure Equation and the SPH}

In the neutron slowing-down domain around the resonance energy, it is supposed that there is no fission and the neutron-nucleus scattering is primarily elastic scattering. Therefore, the neutron source in a region $i$ can be defined as

$$
R_{0 i} \Phi_{i}(u)+R_{1 i} \Phi_{i}(u),
$$

where $R$ is the scattering operator and the indexes 0 and 1 stand for resonant and moderator isotopes, respectively. By factorizing the flux into a product of a constant macroscopic flux $\chi_{i}(u)=\chi(u)$ and a local fine-structure factor $\phi_{i}(u), \Phi_{i}(u)=\chi_{i}(u) \phi_{i}(u)$, and applying the finestructure assumption [8], we obtain the collision-probability based fine-structure equation,

$$
\Sigma_{i}(u) V_{i} \phi_{i}(u)=\sum_{j} P_{i j}(u) V_{j}\left[N_{0 j} r_{0 j} \phi_{j}(u)+\Sigma_{s 1 j}(u)\right],
$$

where $r_{0 i}=R_{0 i} / N_{0 i}$ and $N_{0}$ is the number density of the resonant isotope.

The subgroup method is assumed to apply to a fine energy grid. Using the "Toutes Résonances" (TR) approximation [8], we have

$$
\left(r_{0} \phi\right)_{g} \approx\left(r_{0} \phi\right)_{g}^{T R}=\sum_{g^{\prime}}\left(r_{0} \phi\right)^{g^{\prime} \rightarrow g}
$$

where $\left(r_{0} \phi\right)^{g^{\prime} \rightarrow g}=\sum_{x} a_{x} p_{x}^{g^{\prime} \rightarrow g} \tau_{s 0 x, g^{\prime}}$, and the resonant scattering rate $\tau_{s 0 x, g}=\frac{1}{\Delta u^{g}} \int_{g} d u \sigma_{s 0 x}(u) \phi(u)$. $a_{x}=N_{0 x} / N_{0}$ is the number density proportion of resonant isotope $x$ in the mixture, $p^{g^{\prime} \rightarrow g}$ is the probability for a neutron to scatter from group $g^{\prime}$ to group $g$ after the collision. 
The mathematical probability tables [8] are employed to compute the group-averaged quantities. For a mixture containing $X$ resonant isotopes, the probability table is

$$
\left\{p_{k}, \sigma_{t, k}, \sigma_{\rho x, k}, k=1, \ldots, K, x=1, \ldots, X\right\},
$$

where $p, \sigma_{t}$ and $\sigma_{\rho x}$ are the probability, the total cross section and the partial cross section for reaction $\rho$ and isotope $x$, respectively. $K$ is the order of the probability table.

Finally, the fine-structure-assumption based equation is written for a subgroup $k$ in group $g$ as

$$
\left[\Sigma_{t 1 i}+N_{0 i} \sigma_{t, k}\right] V_{i} \phi_{i, k}=\sum_{j} P_{i j, k} V_{j}\left[N_{0 j} \sum_{x} a_{x} p_{x}^{g \rightarrow g} \sum_{l=1}^{K} p_{l} \sigma_{s 0 x, l} \phi_{j, l}+N_{0 j} \sum_{g^{\prime} \neq g}\left(r_{0} \phi\right)_{j}^{g^{\prime} \rightarrow g}+\Sigma_{s 1 j}\right] .
$$

The Improved Direct Method (IDM) [1,4] is employed to solve Eq. (5). We obtain the region-wise group-averaged flux and reaction rates. By using the mixture probability tables and the TR model, the IDM is capable to solve Eq. (5) with consideration of resonance interference and resonance up-scattering.

Due to the angular dependence issue of multigroup cross sections, the cross sections condensed by the scalar flux can not reproduce the reference results. In consequence, the SPH was applied to the subgroup solution to obtain the multigroup cross sections that preserve the subgroup reaction rates. The detail presentation of the SPH is given in Ref. [4].

\subsection{The Equivalent Dancoff-Factor Cell Method}

As presented in the paper [4], the SG+SPH method gives results in good agreement with Monte Carlo references. However, due to the employment of the mixture treatment and the collision probability calculations using the MCA approximation, the running time can become prohibitive for an assembly depletion calculation.

The Equivalent Dancoff-factor Cell (EDC) method [6] transforms a 2D geometry into a set of 1D cylindrical cells by preserving the 2D Dancoff factors, the EDCs, and carries out self-shielding calculations only on the EDCs. Since the collision probability calculations of 1D cylindrical cells are much faster than those of a 2D geometry, we can expect a drastic reduction in the CPU time of the self-shielding calculation.

The EDC method in APOLLO3 employs the collision probabilities to determine the EDCs. In the Dancoff factor calculations, the fuel cross sections are set to a large value, $10^{5} \mathrm{~cm}^{-1}$, and those of the moderator and the cladding are set to the potential scattering sections.

Suppose that a 2D lattice system has a set of fuel pins, $F_{l}, l=1, L$. The Dancoff factor for pin $F_{l}$ is defined as

$$
D_{F_{l}}^{2 D}=\frac{P_{e s c, F_{l}}^{2 D}}{P_{e s c, F_{l}}^{i s o l}}=\frac{1-P_{F \leftarrow F_{l}}^{2 D}}{1-P_{F_{l} \leftarrow F_{l}}^{i s o l}} .
$$

where $P_{e s c, F_{l}}$ is the fuel escape probability of pin $F_{l}$ defined as the probability that a neutron born uniformly in the fuel pin makes its first collision in moderator or cladding. The index $2 D$ stands 
for the 2D lattice system and the index $i s o l$ stands for the situation in which an isolated fuel pin is surrounded by an infinite homogeneous moderator medium. $F=\sum_{l=1}^{L} F_{l}$, stands for the set of fuel pins in the geometry. The $2 \mathrm{D}$ collision probabilities are computed using the CP-MCA option.

To each fuel pin $F_{l}$, we associate a 1D cylindrical isotropically-reflected cell with the same pin with an external moderator radius $R_{l}$. Its Dancoff factor is defined as

$$
D_{F_{l}}^{1 D}\left(R_{l}\right)=\frac{P_{e s c, F_{l}}^{1 D}\left(R_{l}\right)}{P_{e s c, F_{l}}^{i s o l}}=\frac{1-P_{F_{l} \leftarrow F_{l}}^{1 D}\left(R_{l}\right)}{1-P_{F_{l} \leftarrow F_{l}}^{i s o l}},
$$

using the $1 \mathrm{D}$ cylindrical collision probability calculations with the same cross sections employed in the 2D calculation. To obtain the 1D cylindrical pin that preserves the Dancoff factor in the 2D geometry, from Eqs. (6)(7), we only need to solve the equation

$$
P_{F_{l} \leftarrow F_{l}}^{1 D}\left(R_{l}\right)=P_{F \leftarrow F_{l}}^{2 D} .
$$

The solution is obtained by a binary search. The 1D EDCs need to be calculated once and for all. The self-shielding calculations are applied to these EDCs.

\section{NUMERICAL RESULTS}

We compare the self-shielding methods, the MCA-based and EDC-based SG+SPH methods and the FSM, in PWR fuel cell and fuel assembly calculations. The APOLLO3 flux calculations were carried out by the 2D TDT method of characteristics (MOC) solver. The anisotropic order was set to $\mathrm{P}_{3}$ when using the $\mathrm{SG}+\mathrm{SPH}$ methods, and it was set to $\mathrm{P}_{3}$ or $\mathrm{P}_{0}$ with transport correction $\left(\mathrm{P}_{0} \mathrm{c}\right)$ when using the FSM. The subgroup methods employed the LWR 383-group energy mesh and the mixture treatment; the self-shielding was applied to groups 43 to 351, covering the energy domain from $111.535 \mathrm{keV}$ to $0.55549 \mathrm{eV}$. The FSM options employed the SHEM 281-group energy mesh and the mixture treatment; the self-shielding was applied to groups 43 to 93 , covering the energy domain from $49.9159 \mathrm{keV}$ to $22.5243 \mathrm{eV}$, which is the recommended self-shielding range for using the FSM.

Using the TR scattering model, both the SG+SPH method and the FSM are capable to take into account the resonant up-scattering. Our preliminary tests showed equivalent precision in calculations with or without the consideration of up-scattering. Due to the paper length limit, only the results without considering the up-scattering are given in the following. In consequence, the reference TRIPOLI-4 Monte Carlo calculations employed the traditional SVT (Sampling of the velocity of the target nucleus) model [9]. The TRIPOLI-4 and APOLLO3 calculations were carried out using the CEA V514 library, which is based on JEFF-3.1.1 nuclear data evaluation [10].

\subsection{PWR Fuel Cell Calculations}

Two typical PWR UOX and MOX cells were selected for the preliminary tests. The geometry and material compositions of the two cells are defined in Ref. [4]. For self-shielding calculations, the fuel pin was subdivided into 10 rings, corresponding to $20 \%, 20 \%, 10 \%, 10 \%, 10 \%, 10 \%, 5 \%$, $5 \%, 5 \%, 5 \%$ of the fuel volume, respectively. For flux calculations, each of the 10 fuel rings was further subdivided into eight sectors. 
Table 1 shows the $k_{\text {eff }}$ discrepancies in both UOX and MOX cell calculations. The MCA and EDC methods have obtained similar results, the $k_{\text {eff }}$ errors are less than $80 \mathrm{pcm}$ compared with the references. The FSM $\mathrm{P}_{3}$ option underestimates the $k_{\text {eff }}$ up to $-280 \mathrm{pcm}$. The FSM $\mathrm{P}_{0} \mathrm{c}$ option predicts rather well the $k_{\text {eff }}$ value.

Table 1: PWR fuel cell $k_{\text {eff }}$ discrepancies

\begin{tabular}{llllll}
\hline & \multicolumn{3}{c}{ UOX cell } & & \multicolumn{2}{c}{ MOX cell } \\
\cline { 2 - 3 } \cline { 5 - 6 } Option & $k_{\text {eff }}$ & $\Delta k_{\text {eff }}(\mathrm{pcm})$ & & $k_{\text {eff }}$ & $\Delta k_{\text {eff }}(\mathrm{pcm})$ \\
\hline MCA P $_{3}$ & 1.26950 & 36 & 1.09551 & -66 \\
EDC P $_{3}$ & 1.26940 & 26 & & 1.09543 & -74 \\
FSM P $_{3}$ & 1.26683 & -231 & & 1.09337 & -280 \\
FSM P $_{0} \mathrm{c}$ & 1.26855 & -59 & & 1.09662 & 45 \\
\hline TRIPOLI-4 & $1.26914 \pm 5 \mathrm{pcm}$ & & $1.09617 \pm 5 \mathrm{pcm}$ \\
\hline
\end{tabular}

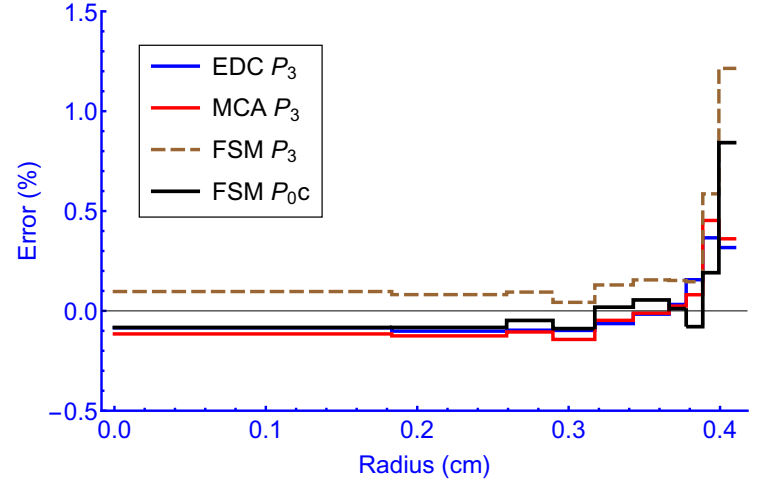

(a) Absorption in UOX cell

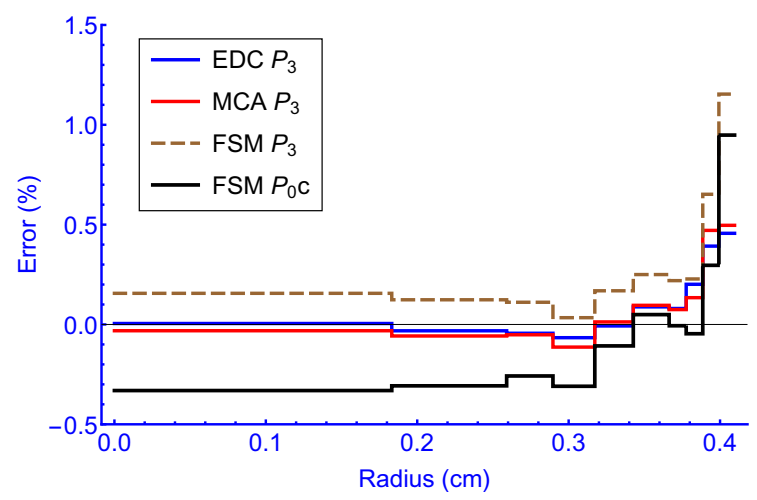

(c) Absorption in MOX cell

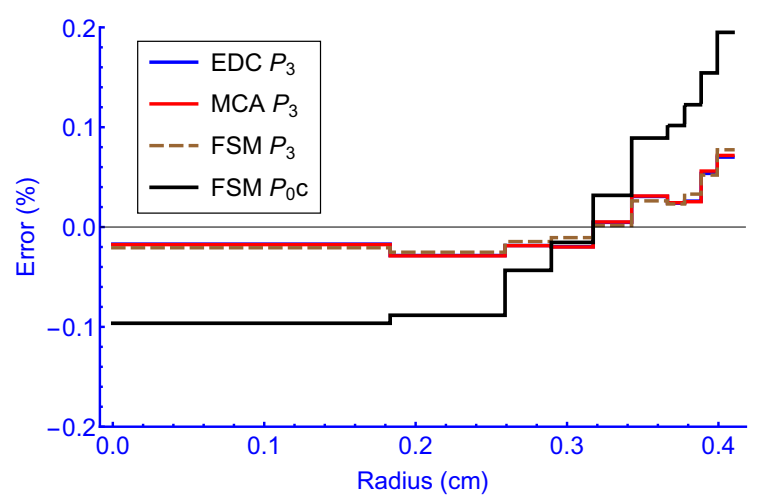

(b) Production in UOX cell

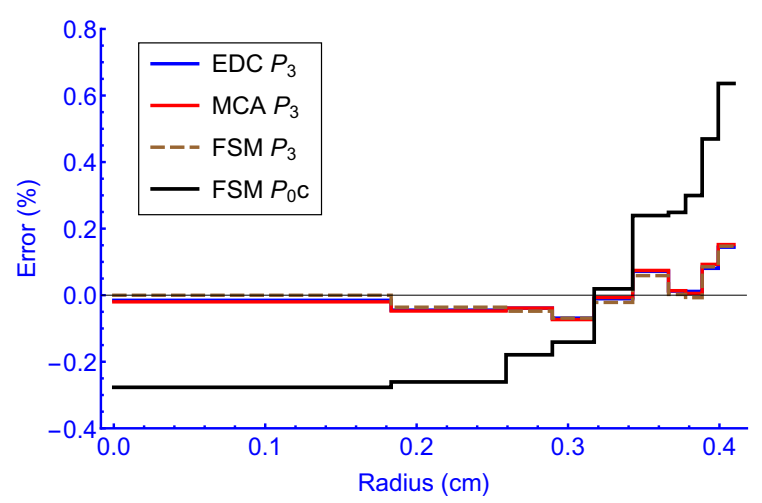

(d) Production in MOX cell

Figure 1: The per-ring reaction rate errors in fuel cells 
Fig. 1 gives the spatial discrepancies in the reaction rates. The MCA and EDC options of the $\mathrm{SG}+\mathrm{SPH}$ method result in a slight underestimation in the inner rings and a slight overestimation in the outer rings. The errors are less than $0.5 \%$ in absorption rates and less than $0.1 \%$ in production rates. This explains why the MCA and EDC methods predicts well the multiplication factors.

The FSM $\mathrm{P}_{3}$ option overestimates the absorption in all rings, whereas it estimates well the production. Therefore, the multiplication factors are underestimated. With the FSM $\mathrm{P}_{0} \mathrm{c}$ option, the underestimation in inner rings and the overestimation in outer rings are worsened in both absorption and production. However, the errors in absorption and production are compensated and the multiplication factors are in better agreement with the references compared with the FSM $\mathrm{P}_{3}$ option.

\subsection{PWR Fuel Assembly Calculation}

We chose to calculate the PWR $\mathrm{UO}_{2}$ assembly defined in the LWR benchmark problem suit [11]. Since it has five Gd-bearing $\mathrm{UO}_{2}$ cells, it is named "Gd-UOX assembly" hereafter. The calculations were carried out at burnup 0 with hot and $0 \%$ void fraction conditions. To avoid the interpolation of nuclear data in both TRIPOLI-4 and APOLLO3 calculations, the temperatures were set to 974 $\mathrm{K}$ for fuel and $574 \mathrm{~K}$ for structural material and moderator. The calculations were carried out on the eighth assembly. In the self-shielding calculations, the fuel pins were subdivided into 10 rings using the same dividing scheme employed in Section 3.1. In the flux calculations, each of the 10 rings was further subdivided into eight sectors. The calculations were carried out by using the CEA V514 library based on JEFF-3.1 nuclear data evaluation [10].

Only the FSM $\mathrm{P}_{0} \mathrm{c}$ option presents large error in $k_{\text {eff }}$ (Table 2), while the three others agree well with the reference. Figs. 2 and 3 presents the errors in spatial absorption and production rates, where the omitted results of the MCA method are similar to those of the EDC method. The results of the EDC and MCA methods agree well to the references, with maximum errors of $0.26 \%$ in absorption rates and $0.35 \%$ in production rates.

With the FSM $\mathrm{P}_{3}$ option, both absorption and production rates are underestimated in the Gd-UOX cells and overestimated in the UOX cells. The good agreement in $k_{\text {eff }}$ is obtained by error compensation. With the FSM $\mathrm{P}_{0} \mathrm{c}$ option, the underestimation in the Gd-UOX cells is worsened, while the overestimation in the UOX cells is reduced, compared with those of the FSM $\mathrm{P}_{3}$ option. The errors in absorption and production are not completely canceled out and they result in a larger $k_{\text {eff }}$ error.

Table 2 also gives the CPU times in self-shielding calculations. The EDC method reduces the CPU time by a factor of 38 compared with the MCA method, while keeping similar precision in $k_{\text {eff }}$ and reaction rates. This fact demonstrates the precision and efficiency of the EDC method.

\section{CONCLUSIONS}

We have compared the newly developed EDC-based SG+SPH method with the FSM method in the PWR fuel cell and assembly calculations. The results show that both EDC-based and MCA-based $\mathrm{SG}+\mathrm{SPH}$ methods are precise in reaction rates and therefore precise in multiplication factors. The 
Table 2: PWR Gd-UOX assembly $k_{\text {eff }}$ discrepancies and CPU times

\begin{tabular}{lllll}
\hline Option & $k_{\text {eff }}$ & $\Delta k_{\text {eff }}(\mathrm{pcm})$ & Mixture-PT (s) & Self-Shield (s) \\
\hline MCA P $_{3}$ & 1.12961 & -51 & 27 & 798 \\
EDC P $_{3}$ & 1.12978 & -34 & 29 & 21 \\
FSM P $_{3}$ & 1.13018 & 6 & 34 & 92 \\
FSM P $_{0} \mathrm{c}$ & 1.13322 & 310 & 35 & 91 \\
\hline TRIPOLI-4 & $1.13012 \pm 2 \mathrm{pcm}$ & & \\
\hline
\end{tabular}

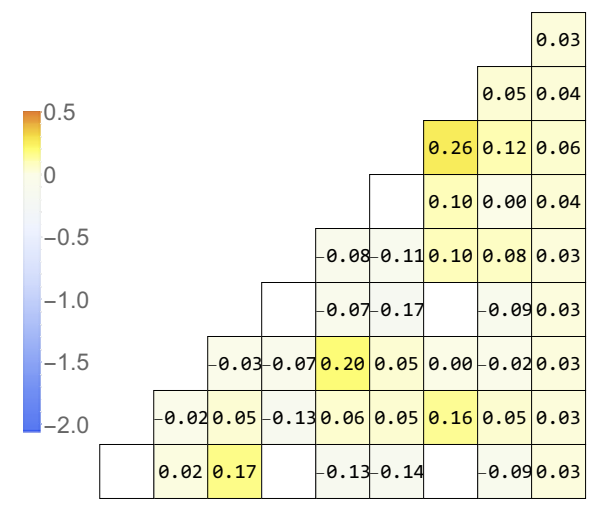

(a) EDC $\mathrm{P}_{3}$

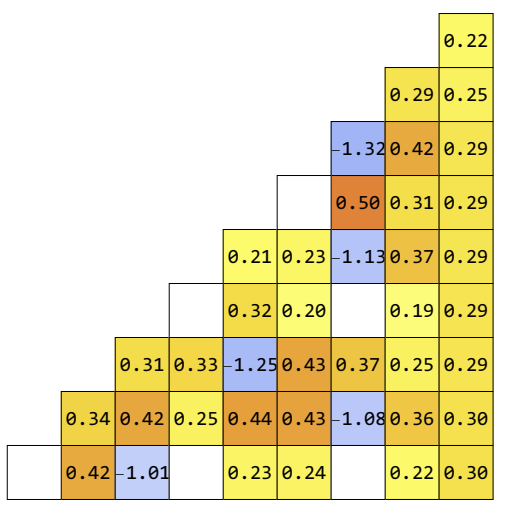

(b) $\mathrm{FSM} \mathrm{P}_{3}$

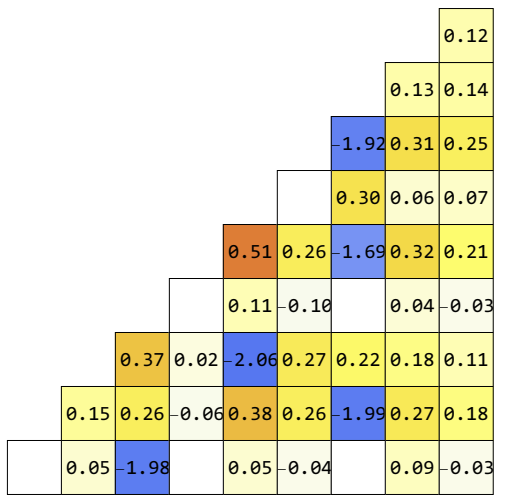

(c) FSM $\mathrm{P}_{0} \mathrm{c}$

Figure 2: The pin-wise absorption rate percent errors in the Gd-UOX assembly

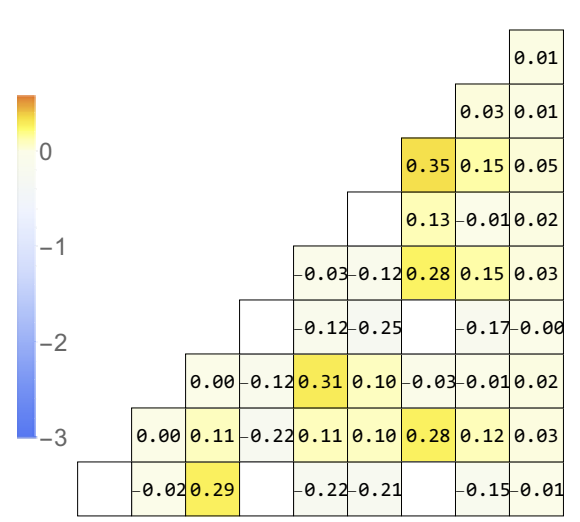

(a) $\mathrm{EDC}_{3}$

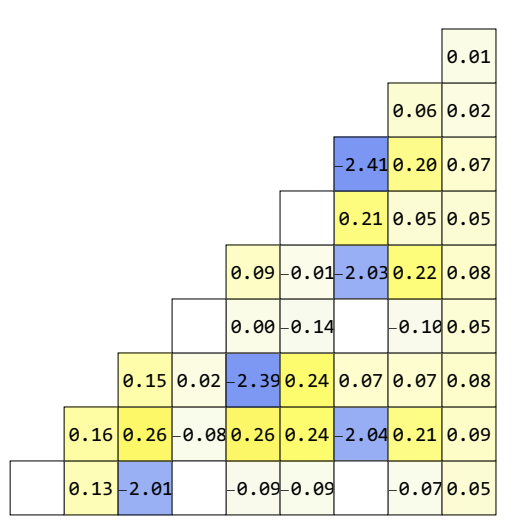

(b) $\mathrm{FSM} \mathrm{P}_{3}$

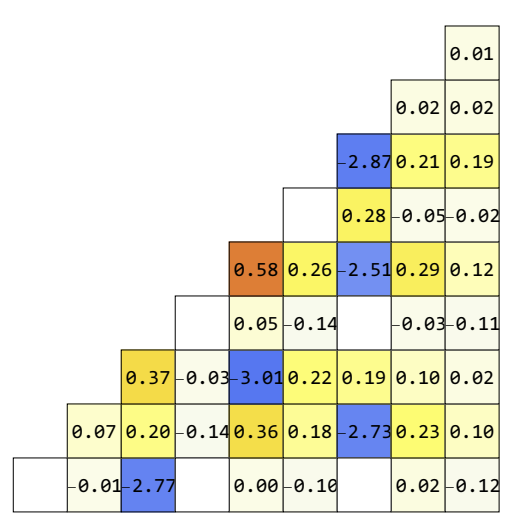

(c) FSM $\mathrm{P}_{0} \mathrm{c}$

Figure 3: The pin-wise production rate percent errors in the Gd-UOX assembly

EDC method is much more efficient than the MCA method by reducing the CPU time by a factor 
of 38 in the Gd-UOX assembly calculation.

With the FSM $\mathrm{P}_{3}$ or $\mathrm{P}_{0} \mathrm{c}$ options, we obtained larger discrepancies in absorption or production rates than with the SG+SPH methods. This proves that the spatial reaction rates are not preserved by the FSM. Sometimes we may obtain a multiplication factor in good agreement with the reference, however it is due to the error compensation.

\section{ACKNOWLEDGEMENTS}

APOLLO $3{ }^{\circledR}$ and TRIPOLI- $4{ }^{\circledR}$ are registered trademarks of CEA. We gratefully acknowledge EDF and Framatome for their long term partnership and their support.

\section{REFERENCES}

[1] L. Mao, R. Sanchez, and I. Zmijarevic. "Considering the up-scattering in resonance interference treatment in APOLLO3 ${ }^{\circledR}$." In $M \& C$ 2015. Nashville, Tennessee, USA, April 19-23, 2015 .

[2] R. Sanchez, I. Zmijarevic, M. Coste-Delclaux, E. Masiello, S. Santandrea, E. Martinolli, L. Villate, N. Schwartz, and N. Guler. "APOLLO2 Years 2010.” Nuclear Engineering and Technology, volume 42(5), p. 474 (2010).

[3] W. Boyd, N. Gibson, B. Forget, and K. Smith. "An analysis of condensation errors in multigroup cross section generation for fine-mesh neutron transport calculations." Annals of $\mathrm{Nu}$ clear Energy, volume 112, pp. 267 - 276 (2018).

[4] L. Mao, I. Zmijarevic, and K. Routsonis. "Application of the SPH method to account for the angular dependence of multigroup resonant cross sections in thermal reactor calculations." Annals of Nuclear Energy, volume 124, pp. 98 - 118 (2019).

[5] A. Hébert and G. Mathonnière. "Development of a Third-Generation Superhomogénéisation Method for the Homogenization of a Pressurized Water Reactor Assembly." Nuclear Science and Engineering, volume 115(2), pp. 129-141 (1993).

[6] Z. Liu, Q. He, T. Zu, L. Cao, H. Wu, and Q. Zhang. "The pseudo-resonant-nuclide subgroup method based global-local self-shielding calculation scheme." Journal of Nuclear Science and Technology, volume 55(2), pp. 217-228 (2018).

[7] L. Mao, I. Zmijarevic, and R. Sanchez. "A subgroup method based on the equivalent Dancofffactor cell technique in APOLLO3 ${ }^{\circledR}$ for thermal reactor calculations." Annals of Nuclear Energy, volume 139, p. 107212 (2020).

[8] M. Coste-Delclaux. "Modélisation du phénomène d'autoprotection dans le code de transport multigroupe APOLLO2." Technical Report CEA-R-6114, Commissariat à l'Energie Atomique (2006).

[9] A. Zoia, E. Brun, C. Jouanne, and F. Malvagi. "Doppler broadening of neutron elastic scattering kernel in Tripoli-4 ${ }^{\circledR}$." Annals of Nuclear Energy, volume 54, pp. 218 - 226 (2013).

[10] A. Santamarina et al. "The JEFF-3.1.1 Nuclear Data Library, JEFF Report 22 Validation Results from JEF-2.2 to JEFF-3.1.1." Technical Report NEA n ${ }^{\circ} 6807$, NEA.

[11] A. Yamamoto, T. Ikehara, T. Ito, and E. Saji. "Benchmark Problem Suite for Reactor Physics Study of LWR Next Generation Fuels." Journal of Nuclear Science and Technology, volume 39(8), pp. 900-912 (2002). 